\title{
Cirripedia Balanomorpha del estuario del Río Paripe (Isla de Itamaracá, Pernambuco, Brasil)
}

\author{
Cristiane Maria Rocha Farrapeira ${ }^{1,2}$ \\ ${ }^{1}$ Laboratório de Cirripedia, Departamento de Biologia, \\ Universidade Federal Rural de Pernambuco - UFRPE, \\ Rua D. Manoel de Medeiros, s/n, Dois Irmãos, CEP 52171-900, Recife, PE, Brasil \\ ${ }^{2}$ Autor para correspondencia: Cristiane Maria Rocha Farrapeira, e-mail: c.farrapeira@db.ufrpe.br
}

FARRAPEIRA, C.M.R. 2008. Cirripedia Balanomorpha of Paripe River estuary (Itamaracá Island, Pernambuco, Brazil). Biota Neotrop. 8(3): http://www.biotaneotropica.org.br/v8n3/en/ abstract?article+bn00208032008.

\begin{abstract}
Barnacles are animals characteristic and abundant of intertidal region on marine and estuarine environments worldwide, but they are little known in the areas of tropical mangroves. This study was done with the aim to know the barnacles' species of the estuary of the Paripe River (Pernambuco, Brazil), indicating some characteristics of its distribution throughout the estuary. In dry season of 2006, the animals were collected in three stations of the estuary, in all hard substrata available, observing their vertical distribution. In polihaline station (salinities varying from 28.7 to 36.1), it was found eight species: Amphibalanus amphitrite, A. improvisus, A. reticulatus, A. venustus, Chthamalus proteus, C. bisinuatus, Microeuraphia rhizophorae and Striatobalanus amaryllis. The species classified as euryhaline, found until the mesohaline region of the estuary (salinities varying from 10.8 to 19.6) were: Amphibalanus amphitrite, A. improvisus, A. reticulatus, Chthamalus proteus and Microeuraphia rhizophorae, whereas in the oligohaline region (salinities varying from 0.2 to 5.2), only M. rhizophorae was present, being classified as estuarine component. A horizontal zonation of species of barnacles was clearly observed from the mouth until the upstream, with gradual decrease of number of species and it was confirmed their pattern of vertical distribution in relation to the height of tides, according with its respective ecological valences. The introduced exotic species in Brazil, Amphibalanus reticulatus and Striatobalanus amaryllis, were well adapted on natural and artificial substrata of the estuary.

Keywords: Barnacle, estuary, distribution, tropical mangroves.
\end{abstract}

FARRAPEIRA, C.M.R. 2008. Cirripedia Balanomorpha del estuario del Río Paripe (Isla de Itamaracá, Pernambuco, Brasil). Biota Neotrop. 8(3): http://www.biotaneotropica.org.br/v8n3/es/ abstract?article+bn00208032008.

Resumen: Los cirrípedos son animales característicos y abundantes en la región intermareal de los ambientes marinos y estuarinos de todo el mundo, pero son poco conocidos en las áreas de manglares tropicales. El presente estudio fue realizado con el objetivo de conocer las especies de cirrípedos en el estuario del Río Paripe (Pernambuco - Brasil), señalando algunas características de su distribución a lo largo del estuario. En la estación de estiaje de 2006, los animales fueron colectados en tres estaciones del estuario, en todos los substratos duros disponibles, observando su distribución vertical en los mismos. En la estación polihalina (salinidades variando de 28,7 hasta 36,1), se encontraron ocho especies: Amphibalanus amphitrite, A. improvisus, A. reticulatus, A. venustus, Chthamalus proteus, C. bisinuatus, Microeuraphia rhizophorae y Striatobalanus amaryllis. Las especies clasificadas como eurihalinas, encontradas hasta la región mesohalina del estuario (salinidades variando de 10,8 hasta 19,6) fueran: Amphibalanus amphitrite, A. improvisus, A. reticulatus, Chthamalus proteus y Microeuraphia rhizophorae, mientras que en la región oligohalina (salinidades variando de 0,2 hasta 5,2), solamente M. rhizophorae estuvo presente, sendo por tanto, clasificada como componente estuarino. Una zonación horizontal de especies de cirrípedos fue claramente observada a partir de la desembocadura hasta al montante del río, con disminución gradual del número de especies y fue confirmado su padrón de distribución vertical en relación a las alturas de mareas, observadas las respectivas valencias ecológicas. Las especies exóticas introducidas en Brasil, Amphibalanus reticulatus y Striatobalanus amaryllis, estaban bien adaptadas en los substratos naturales y artificiales del estuario.

Palabras clave: Cirrípedos, estuario, distribución, manglares tropicales. 


\section{Introducción}

Los cirrípedos comprenden un grande número de crustáceos sesiles presentes en todos los océanos y climas del mundo (Newman \& Ross 1976), sendo un grupo bien sucedido en términos de abundancia y diversidad (Marchinko et al. 2004). Existen diversas especies que toleran o requieren condiciones estuarinas; en este caso ellas son dominantes en el ambiente, en conjunto con las ostras y mejillones (Garduño 1980, Inclán 1989, Rivadeneyra 1989, Farrapeira 2006).

Hay una vasta literatura sobre éste grupo de animales, abordando diversos aspectos como taxonomía, ecología y fisiología, refiriéndose a los ambientes marinos y estuarinos, especialmente en regiones de clima templado del mundo. Entretanto, en las regiones tropicales y en áreas de manglares hay pocos estudios. En Brasil, los trabajos que estudiaron específicamente los cirrípedos, investigando su distribución horizontal y vertical en ambientes estuarinos, fueron realizados en los estados de Rio de Janeiro (Oliveira 1941, Lacombe \& Monteiro 1974, Lacombe 1977, Lacombe \& Rangel 1978, Silva-Brum \& Absalão 1989, Silva-Brum \& Martins 1997, Junqueira et al. 2000) y Pernambuco (Farrapeira, dados no publicados, Farrapeira 2006).

Las regiones estuarinas tropicales presentan áreas con considerables diversidades de organismos, donde las variaciones específicas, temporales y espaciales están relacionadas directamente con la influencia de los parámetros ambientales (Gunter, 1961). Barnes (1989) sugirió que hay un empobrecimiento de la diversidad de las especies, guardando relación con el gradiente salino del estuario, ya que las especies que habitan los estuarios son, en su inmensa mayoría, especies marinas, con escasa capacidad de competición ínter-específica pero muy tolerantes, lo que les permite aprovechar habitats marginales al marino, como es el caso de la zona de aguas salobres de los estuarios. Si se avanza siguiendo el cauce del río, partiendo del mar, las aguas salobras son sustituidas progresivamente por las aguas más dulces, ocurriendo gradualmente la sustitución de las comunidades de organismos o el desaparecimiento de ciertas especies, hecho denominado zonación horizontal (Achituv, 1984). Considerando que los cirrípedos son sesiles y viven relativamente durante longo tempo, ellos reflejen las condiciones prolongadas del lugar en donde ocurren y pueden ser utilizados como bioindicadores para distinguir las respuestas generales a una fuente ambiental biodisponible (Al-Thaqafi \& White 1991). De esta forma, el presente estudio tuvo la finalidad de generar información sobre la composición de los cirrípedos del estuario del Río Paripe, Pernambuco, Brasil y su distribución horizontal y vertical a lo largo del estuario, de modo a constituir un material diagnóstico para futuros inventarios y facilitar la comparación con otros ambientes de manglares.

\section{Materiales y Métodos}

\section{Descripción del área}

El estuario del Río Paripe situase al sur de la Isla de Itamaracá, próximo a la Barra Sur del Canal de Santa Cruz, en Pernambuco, Brasil (Figura 1). El estuario es pequeño (1,6 km de longitud), de baja profundidad en las bajamares (máxima de $0,80 \mathrm{~m}$, en el lecho del río) y posee un manglar extenso compuesto por las especies Rhizophora mangle Linnaeus, Avicennia schaueriana Stapf \& Leechman y Laguncularia racemosa (Linnaeus) (Farrapeira et al. 2000). La acción humana en esto ambiente aún es considerada mínima, una vez que la área es poco habitada y a lo largo del estuario no se encuentra ningún empresa industrial o inmobiliario (Monteiro \& Coelho-Filho 2004), encontrándose, por esto motivo, libre de polución (Branco et al. 2003).
En este estuario, la temperatura superficial del agua presenta poca variación anual, alrededor de $4{ }^{\circ} \mathrm{C}$ durante el año, registrándose las temperaturas más bajas en la estación lluviosa (mayo hasta agosto) $\left(25-26^{\circ} \mathrm{C}\right)$ y las máximas $\left(27-31^{\circ} \mathrm{C}\right)$, en la estación de estiaje del año (entre septiembre y abril) (Branco et al. 2003). Su marea dinámica presenta una amplitud máxima en mareas de sicigia y de cuadratura alrededor de 2,5 y $1,8 \mathrm{~m}$ respectivamente, con dados relativos al Puerto de Recife (DHN 2006).

\section{Metodología}

Por medio de GPS fueron demarcadas tres estaciones de muestreo, tomando como base la fisiografía del manglar y condiciones ecológicas: la primera ( $7^{\circ} 48^{\prime} 39^{\prime \prime} \mathrm{S}$ y $34^{\circ} 51^{\prime} 23^{\prime}$ ' O), localizada en la desembocadura del río y ubicada en las márgenes del Canal de Santa Cruz, es bordeada por mangles principalmente de las especies Rhizophora mangle y Avicennia schaueriana, con algunas rocas aisladas presentes en el fondo fangoso somero, una ponte rocosa en ruinas y estacas de madera de una pasarela. La segunda estación ( $7^{\circ} 48^{\prime} 27^{\prime}$ 'S y $34^{\circ} 51^{\prime} 14^{\prime \prime} \mathrm{O}$ ), situada en la parte media del estuario, prójima a una bifurcación del río, presenta tres especies de mangles, $R$. mangle, A. schaueriana y Laguncularia racemosa; en este trecho, las márgenes fangosas y el lecho del río presentan muchas conchas vacías de ostras y mariscos, resultado de la acción de los marisqueros. La tercera y última estación ( $7^{\circ} 47^{\prime} 58^{\prime \prime}$ S y $34^{\circ} 51^{\prime} 22^{\prime \prime}$ O), fue demarcada en la cabecera del río, en el limite de distribución del manglar, en esta región compuesto únicamente por ejemplares enanos de la especie Laguncularia racemosa (Figura 1).

El estudio fue realizado en el periodo de la estación de estiaje de 2006 (de septiembre hasta diciembre). En cada estación, la salinidad del agua fue medida por medio de un refractómetro portátil, en las pleamares y bajamares, durante los cuatro meses estudiados con muestras retiradas de la superficie del agua, en el centro del río. La temperatura fue medida por medio de termómetro común (escala Celsius) solamente en la estación un, durante las pleamares.

Los ejemplares de cirrípedos fueron muestreados mensualmente durante las mareas bajas, sendo retirados de todos sustratos duros disponibles en la zona intermareal e infralitoral raso, representados por las raíces y troncos de árboles de mangle, sustratos rocosos del puente (en ruinas), pilotes y estacas de maderas de la pasarela, rocas esparcidas, así como sobre conchas y caparazones de otros animales bentónicos. Antes de retirar los especimenes de los sustratos fue procedida la medición de sus alturas máximas y mínimas de distribución vertical en relación a los niveles de mareas, usando una cinta métrica graduada en centímetros, tomando por base el nivel mínimo de la bajamar $(0,0 \mathrm{~m})$ y los dados de altura y horarios de la Tabla de Mareas para el Puerto del Recife, informado por DHN (2006), y el limite máximo de distribución de los cirrípedos Chthamalidae, siguiendo la metodología propuesta por Rosa Filho \& Farrapeira-Assunção (1998). Los cirrípedos fueron retirados mediante la utilización de espátula y martillo y los organismos sesiles asociados fueron anotados, para caracterización de la comunidad bentónica.

En el Laboratorio de Cirripedia de la Universidade Federal Rural de Pernambuco- UFRPE los animales fueron fijados en alcohol a $70 \%$ y disecados y analizados con auxilio de estereomicroscópio. La clasificación fue hecha según los trabajos de Young (1991, 1993, 1994) y Farrapeira (2006). Todos los especimenes se encuentran depositados en el referido laboratorio.

Las estaciones del estuario fueron clasificadas en cuanto a la variación de la salinidad según la definición del "Symposium on the Classification of Brackish Waters" en Venecia, 1958 (Rodríguez 1963): Euhalina (40-30), polihalina (30-18), mesohalina (18-05) y oligohalina (05-0.5). Además, los cirrípedos fueron clasificados de acuerdo con su tolerancia a la salinidad, de acuerdo con Day (1967), 

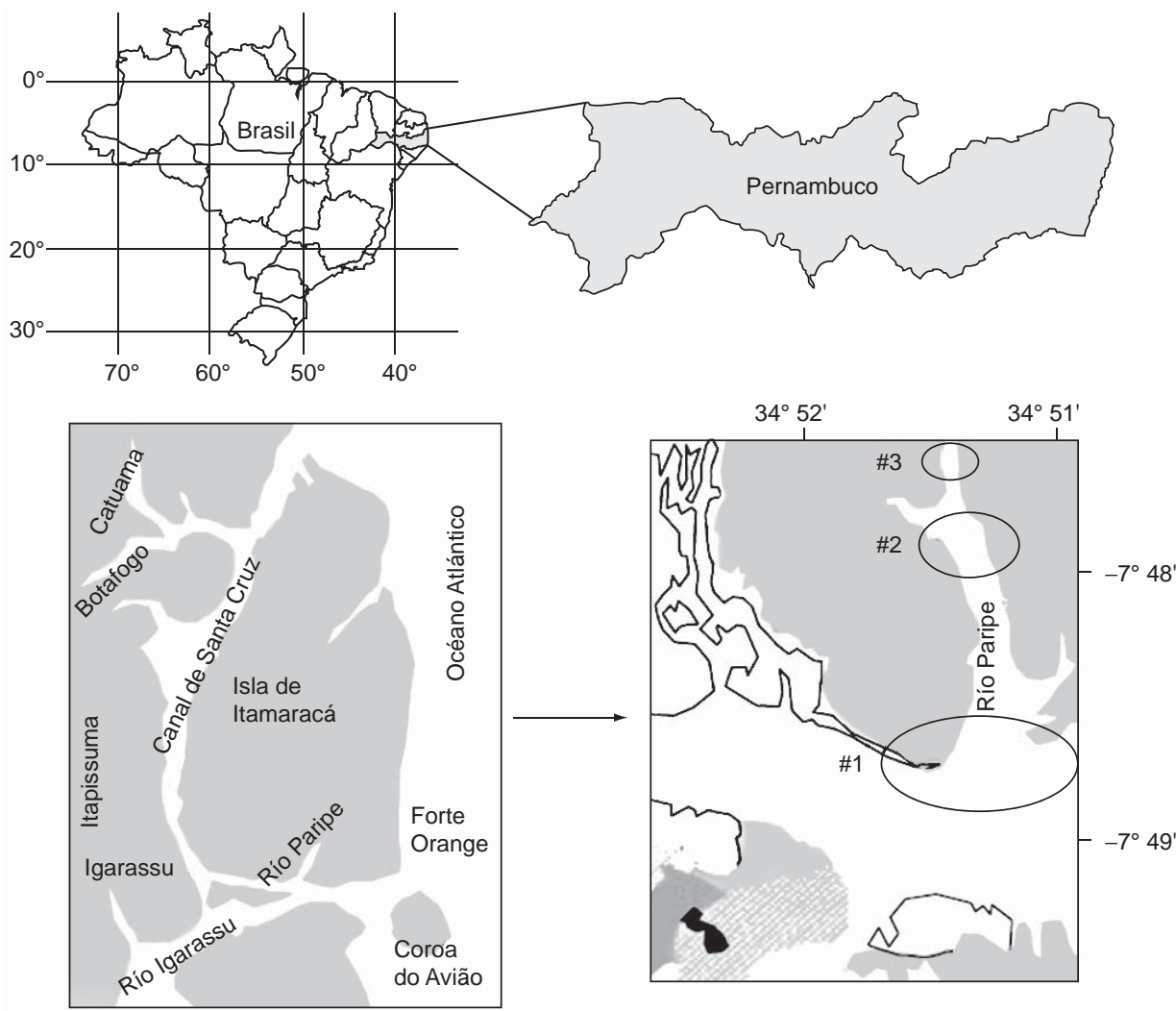

Figura 1. Localización del área de estudio, indicando: Brasil, Pernambuco, Isla de Itamaracá circundada por el Canal de Santa Cruz y estuario del Río Paripe, con las tres estaciones (\#) de muestreo (Adaptado de Branco et al. 2003).

Figure 1. Localization of the studied area, indicating: Brazil Pernambuco, Itamaracá Island surrounded by the Channel of Santa Cruz, and estuary of the Paripe River, with the three sample' stations (\#) (Adapted from Branco et al. 2003).

en componentes: marino estenohalino, restringido a aguas de alta salinidad, cerca de la desembocadura del río (especies que no toleran grandes variaciones de salinidad); marino eurihalino, que se extiende desde el mar a través de todo el estuario (en las zonas de mezcla de las aguas dulces con las marinas con la salinidad alcanzando valores muy variables, bien por lo hipersalino como por lo hiposalino); y componente estuarino, que ha evolucionado de forma marina, pero que se halla ahora restringido a estuarios (especie de aguas tranquilas, nunca encontrada en el mar); en esto último caso, fueron utilizadas también fuentes bibliográficas para establecer el padrón.

\section{Resultados}

La temperatura superficial del agua se mantuvo con poca variación durante el periodo estudiado, variando de 26,5 hasta $30,0^{\circ} \mathrm{C}$. La salinidad del agua tuvo cambios considerables entre las estaciones de colecta y los niveles de mareas (bajamares y pleamares máximas). La estación 1 presentó los valores más altos de salinidad durante todo el período de estudio, variando desde 28,7 (durante la bajamar, en septiembre) hasta 36,1 (en diciembre, durante la pleamar) y fue clasificada como estación euhalina - polihalina. La estación 2 sufrió un poco más de variación: 10,8 hasta 19,6, en las bajamares y desde 19,7 hasta 29,8 , durante las pleamares, teniendo sido clasificada como polihalina - mesohalina. En la estación 3, la salinidad varió poco: desde 0,2 (en la bajamar, en el mes de septiembre) hasta 5,2 (valor máximo, durante una pleamar de diciembre), sendo por esto considerada oligohalina (Figura 2).
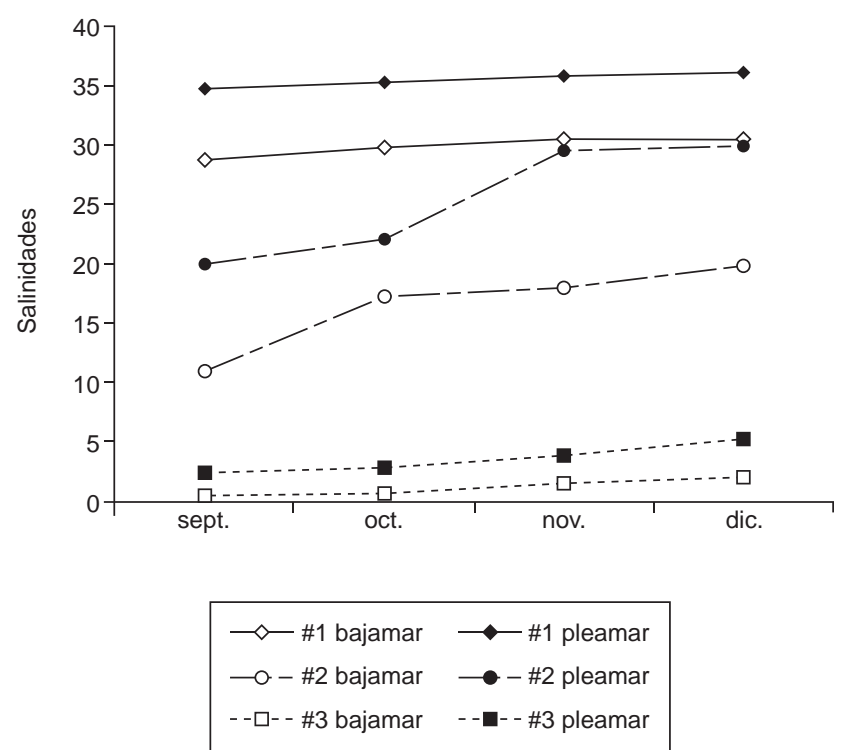

Figura 2. Valores de salinidades de las tres estaciones de muestreo de cirrípedos en el estuario del Río Paripe, Isla de Itamaracá, Brasil, durante las bajamares y pleamares del estación de estiaje de 2006.

Figure 2. Values of the salinities of the three sampling' stations of barnacles in the estuary of the Paripe River, Island of Itamaracá, Brazil, during the low and high tides of the dry season 2006. 
Entre los animales incrustantes encontrados en el estuario del río Paripe, fueron identificados ocho especies de Cirripedia Balanomorpha: Amphibalanus amphitrite (Darwin 1854) (Figura 3), A. improvisus (Darwin 1854) (Figura 4), A. reticulatus (Utinomi 1967) (Figura 5), A. venustus (Darwin 1854) (Figura 6), Chthamalus bisinuatus Pilsbry, 1916 (Figura 7), C. proteus Dando \& Southward, 1980 (Figura 8), Microeuraphia rhizophorae (Oliveira 1940) (Figura 9), y Striatobalanus amaryllis (Darwin 1854) (Figura 10). Los cirrípedos poden ser reconocidos visualmente por sus características morfológicas externas exhibidas en las respectivas figuras e identificados a partir de la clave dicotómica que se siegue.

En la estación 1 fueron encontradas las ocho especies de cirrípedos, destacándose como marinas estenohalinas, encontradas apenas en esta región: Amphibalanus venustus, Chthamalus bisinuatus y Striatobalanus amaryllis. Las especies presentaran una gradación de posición vertical en los sustratos en relación a la altura de mareas.

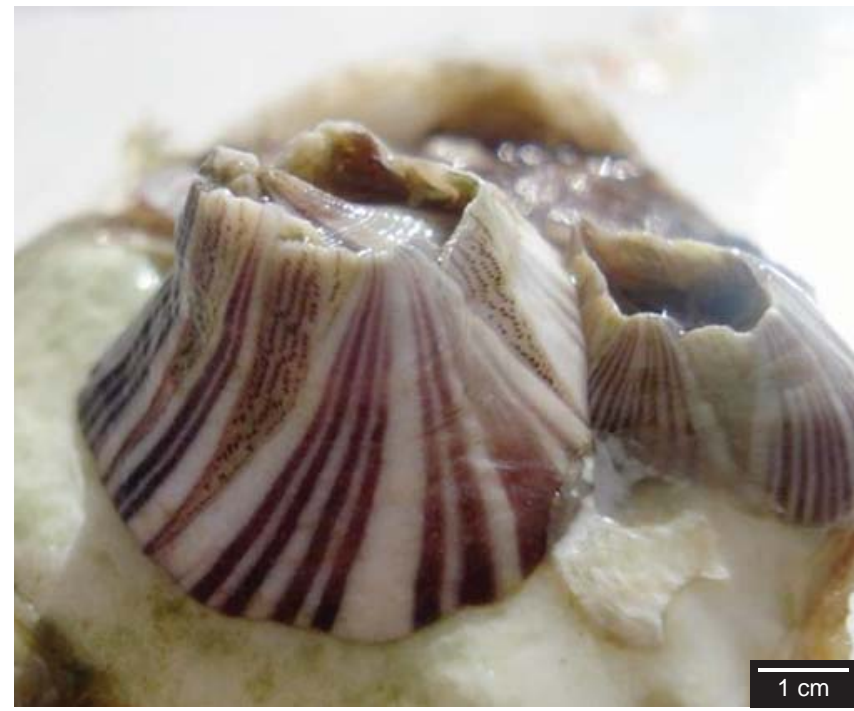

Figura 3. Amphibalanus amphitrite sobre ostra Crassostrea rhizophorae. Figure 3. Amphibalanus amphitrite on oyster Crassostrea rhizophorae.

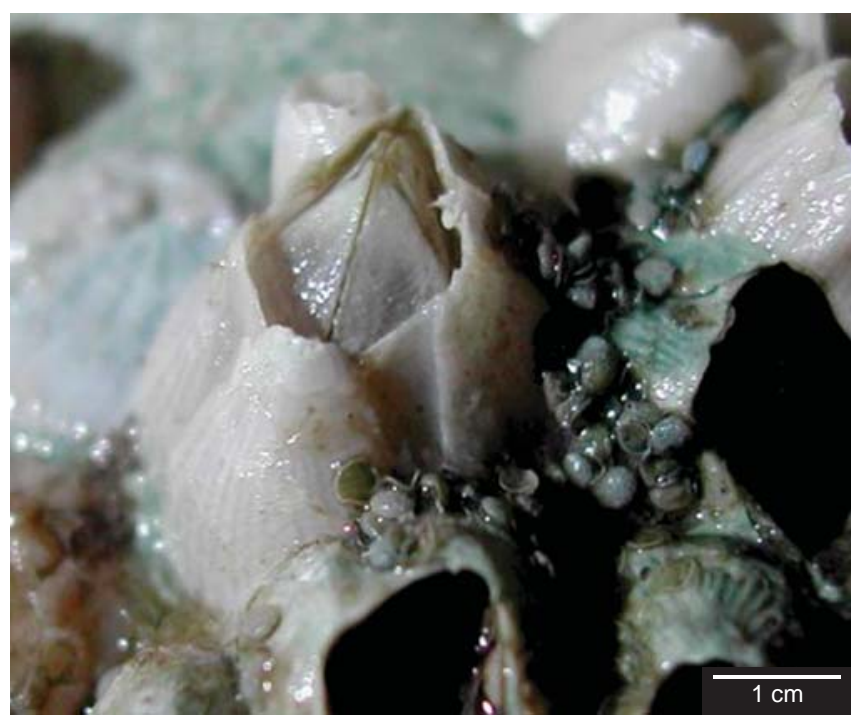

Figura 4. Amphibalanus improvisus sobre ostra Crassostrea rhizophorae. Figure 4. Amphibalanus improvisus on oyster Crassostrea rhizophorae.
Así, los ejemplares de Chthamalus bisinuatus, fijos únicamente sobre sustratos rocosos de la puente en ruinas expuestos al sol, formaran un cinturón de cerca de $20 \mathrm{~cm}$ en la zona intermareal más alta, en el limite con el supralitoral. En la correspondiente región localizada en los mangles, Microeuraphia rhizophorae fue dominante numéricamente, ocupando una área de aproximadamente $60 \mathrm{~cm}$. En la porción media del intermareal, la llamada zona balanoide, fue observada la predominancia numérica de la especie Amphibalanus amphitrite ocupando una porción de 80-120 cm, fijada sobre conchas de ostras Crassostrea rhizophorae Guilding, 1828 juntamente con Chthamalus proteus y algunos raros mejillones Brachidontes exustus Linnaeus, 1758.

En el límite con el infralitoral de la estación 1, los sustratos rocosos (incluyendo las piedras esparcidas) y maderas de los pilotes de la pasarela fueron los únicos disponibles. En este piso, las especies Striatobalanus amaryllis y Amphibalanus reticulatus fueron encontradas abundantemente apenas sobre rocas en el fundo fangoso del Canal

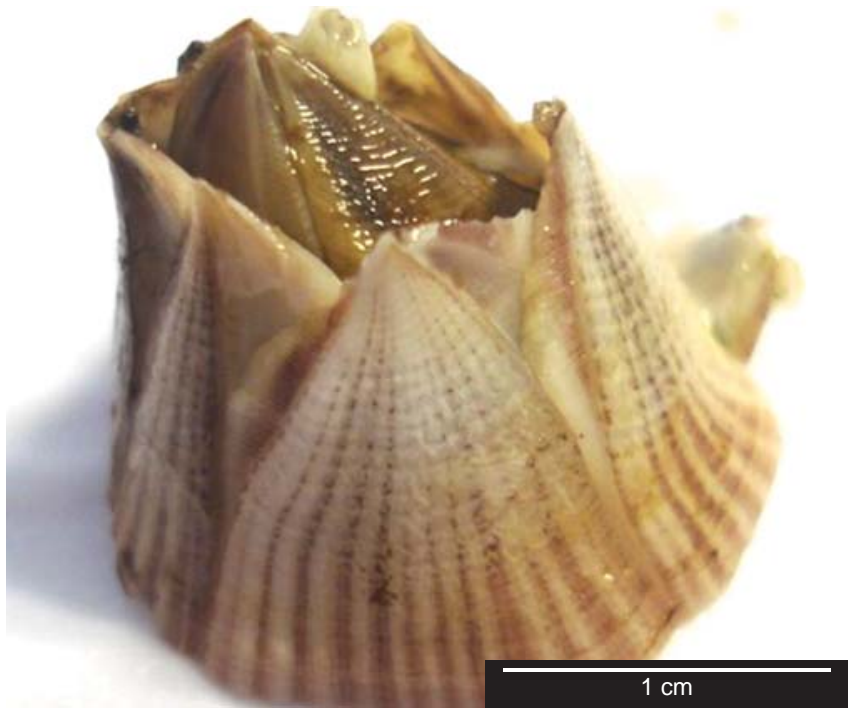

Figura 5. Amphibalanus reticulatus.

Figure 5. Amphibalanus reticulatus.

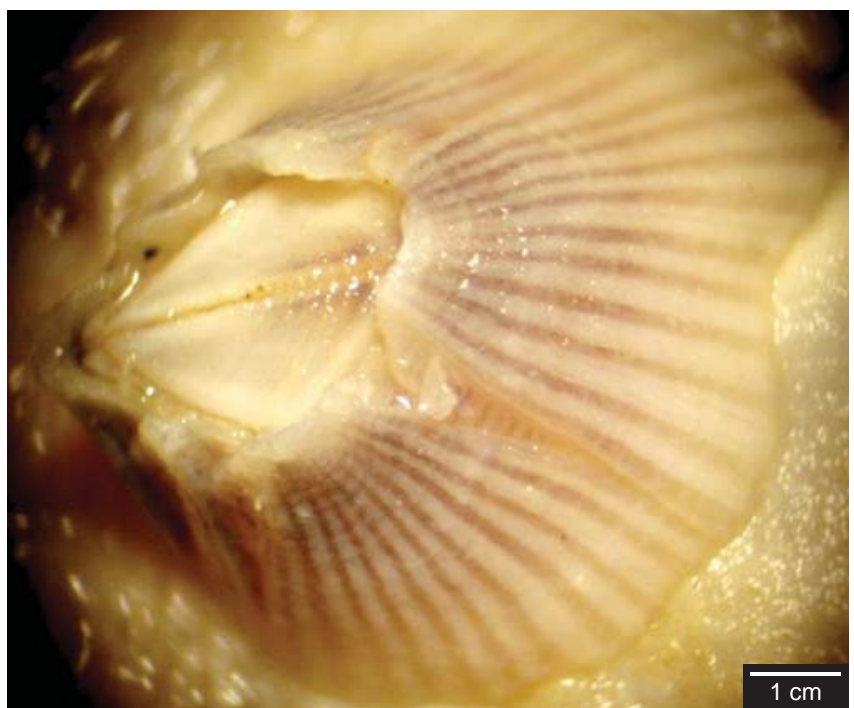

Figura 6. Amphibalanus venustus.

Figure 6. Amphibalanus venustus. 


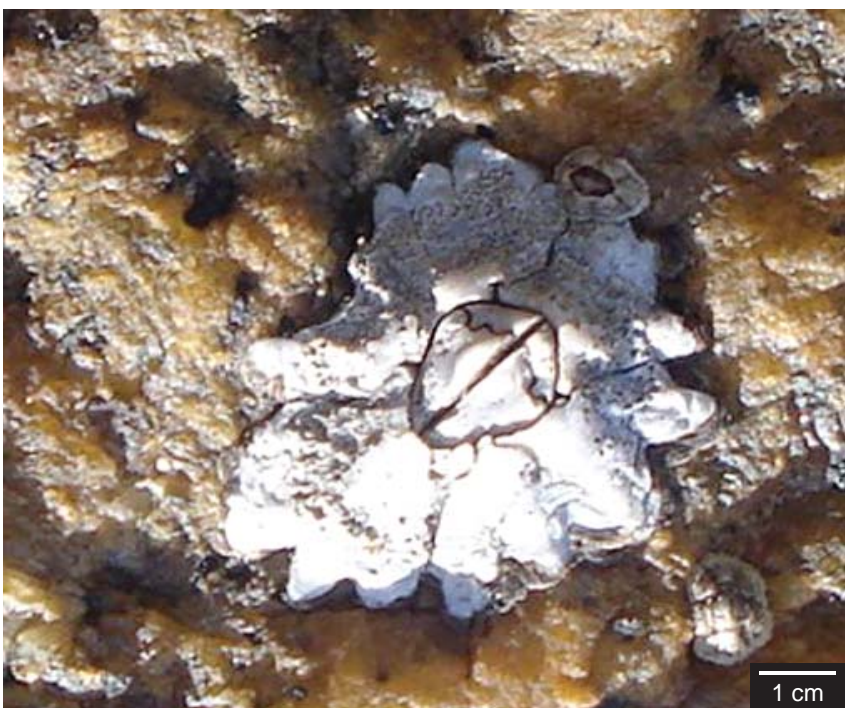

Figura 7. Chthamalus bisinuatus en sustrato rocoso.

Figure 7. Chthamalus bisinuatus on rocky substrate.

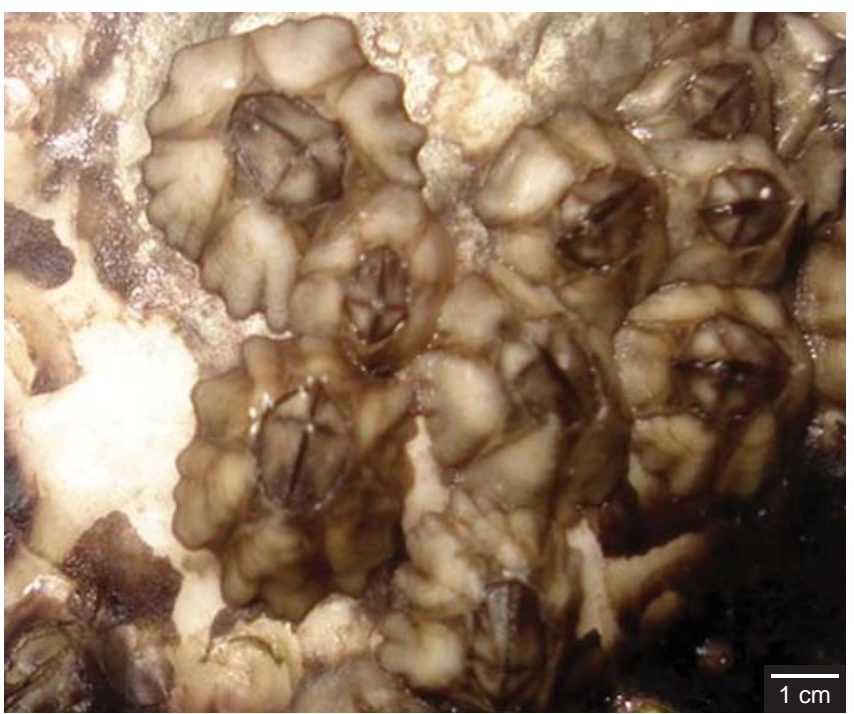

Figura 8. Chthamalus proteus; grupo de ejemplares sobre ostra Crassostrea rhizophorae.

Figure 8. Chthamalus proteus; group of specimens on oyster Crassostrea rhizophorae.

de Santa Cruz y en el paredón rocoso de la puente en ruinas habiendo sido los principales basibiontes de las otras especies de cirrípedos. Algunos ejemplares de $S$. amaryllis estaban casi completamente revestidos por la esponja de fuego Tedania ignis (Porifera) y, sobre sus conchas, fueron comunes las especies epibiontes: Amphibalanus improvisus, A. reticulatus y A. venustus. Esta última fue también encontrada fijada sobre conchas de gasterópodos Cerithium atratum (Born 1778) habitadas por cangrejos ermitaños (Diogenidae) y sobre bivalvos Ostrea equestris (Say 1834) incrustados sobre estas conchas. En el lecho del río, A. improvisus fue también encontrada sobre conchas vacías de ostras y mariscos: Lucina pectinata (Gmelin 1791), Iphigenia brasiliana (Lamark 1818) y Anomalocardia brasiliana (Gmelin, 1791).

En la estación 2 se registró la ocurrencia de apenas cinco especies de cirrípedos, distribuida en troncos de las tres especies de mangles, en un espacio de cerca de 1,90 m, en relación a los niveles máximos

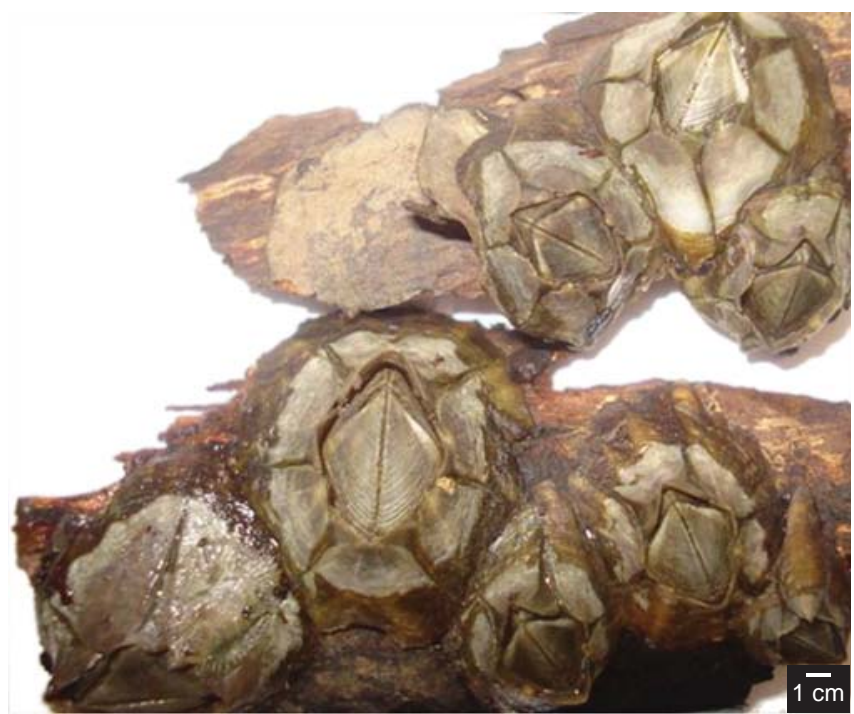

Figura 9. Euraphia rhizophorae; grupos de ejemplares sobre mangle Laguncularia racemosa.

Figure 9. Euraphia rhizophorae; groups of specimens on mangrove Laguncularia racemosa.

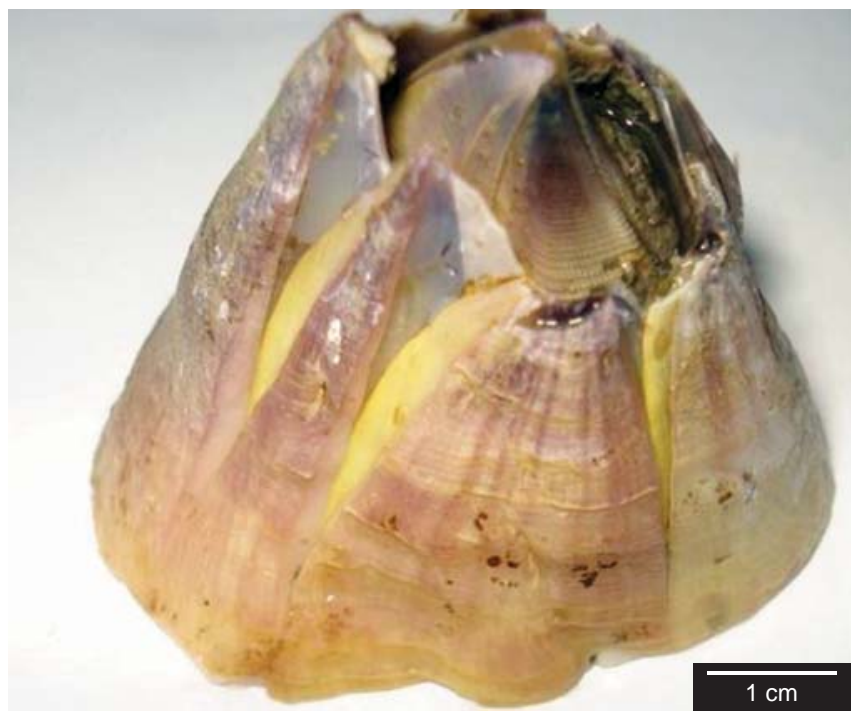

Figura 10. Striatobalanus amaryllis.

Figure 10. Striatobalanus amaryllis.

y mínimos de las mareas. En esta región, el mismo padrón de distribución vertical fue observado para las especies. Microeuraphia rhizophorae estaba fijada en las porciones más altas de los troncos y raíces de mangles de la región intermareal, formando un cinturón de $60 \mathrm{~cm}$ de altura, con raros especimenes de Chthamalus proteus, que también ocurrió hasta el medio del región del intermareal. En la zona balanoide, Amphibalanus amphitrite y la ostra Crassostrea rhizophorae estaban distribuida en cerca de $120 \mathrm{~cm}$, sendo que los $50 \mathrm{~cm}$ inferiores, tuvo mezcla de espacio con A. improvisus y A. reticulatus, en el limite del piso intermareal con el infralitoral. Estas dos especies se fijaron tanto en raíces de mangles, cuanto en conchas vacías de los mariscos: Lucina pectinata, Iphigenia brasiliana y Anomalocardia brasiliana, en el lecho del río. Considerando su distribución en la región estuarina, estas especies fueron consideradas como marinas eurihalinas. 
En la estación 3 había solamente la especie Microeuraphia rhizophorae, fijada en troncos de mangle de Laguncularia racemosa, en un espacio vertical alrededor de $40 \mathrm{~cm}$, presentando relevante ecomorfogénesis, representada por la disminución de talla y desgaste de las placas parietales. Ninguna otra especie de cirrípedo o de otro taxon incrustante fue encontrada. Esta especie por hacer tenido la más amplia distribución en el estuario del Río Paripe, tolerando los más amplios valores de salinidades, fue considerada un componente estuarino.

\section{Discusión}

El empobrecimiento de la diversidad de las especies habitantes de estuarios en el sentido mar - agua dulce sugerida por Achituv (1984) y Barnes (1989), relacionado con el gradiente salino del estuario, fue también verificado, observando una disminución del numero de especies de Cirripedia a partir de la área más marina, en la desembocadura del río, con ocho especies, para cinco, en la región media del estuario e, después, solamente una especie, en la area situada en la cercanía del agua dulce.

Bulger et al. (1993) reconoció que la salinidad no es la única variable importante gobernando la distribución de las especies estuarinas; esto factor actúa cómo una variable sustituta para las características de los hábitats que varían junto con la salinidad (sustrato, turbidez). Sin embargo, efectos directos de la salinidad sobre los organismos poden quedarse responsables por algunos padrones observados en el estuario. La significativa disminución gradual del número de especies de cirrípedos en el sentido desembocadura-montante del río, coincidió con las observaciones de algunos autores brasileños. Correia (1998) observó una disminución de especies bentónicas en relación a estaciones más y menos salinas del ambiente estuarinolagunar de Mundaú-Manguaba, Alagoas, con tres especies en las regiones próximas al mar (Microeuraphia rhizophorae, Amphibalanus amphitrite y A. improvisus) y solamente dos especies (A. improvisus y Fistulobalanus citerosum) en las áreas de menor salinidad. Farrapeira (2006), estudiando la ocurrencia de los cirrípedos en la area estuarina de Recife, Pernambuco, encontró en la región portuaria, polihalina, nueve especies, decreciendo para cinco especies en las áreas de grande variación salina en relación a las mareas, con casi las mismas especies eurihalinas del Río Paripe, a la excepción de Amphibalanus eburneus, que es una especie ampliamente encontrada en las regiones estuarinas de Recife y de otras localidades y considerada típica de estuarios y bahías (Southward 1975, Young 1994).

Cabe un destaque para una diferencia de ocupación de nicho ecológico de cirrípedos en áreas estuarinas de bajas salinidades. En el estuario del Río Paripe, solamente Microeuraphia rhizophorae fue encontrada resistiendo en esta situación ecológica, destacando para el facto de que esto es un estuario de casi ninguna polución (Branco et al. 2003). En contrapartida, Farrapeira (2006) observó que en el área estuarina de Recife A. amphitrite ocupaba esto nicho, estando sometida también a aguas bastante polucionadas con desechos domésticos. Silva-Brum \& Absalão (1989), constataron que las comunidades de cirrípedos de la Bahía de Guanabara (Rio de Janeiro) sufrieron poca diferencia en cuanto a la composición de especies, desde la entrada de la bahía hasta el final, aunque solamente aquellas con baja resistencia a las grandes variaciones de salinidad y/o polución de las aguas no ocurrieron en todas las estaciones. Estos autores observaron, todavía, que ocurrió una creciente tasa de mortalidad de los ejemplares, en el sentido mar-estuario y un correspondiente decrecimiento en la densidad de los organismos, encontrando solamente A. amphitrite en las áreas de mayor polución doméstica. En su estudio sobre la sucesión ecológica de organismos bentónicos en areas de diferentes grados de polución en la misma región, Breves-Ramos et al. (2005) observaran una elevada porcentaje de recubrimiento de los sustratos artificiales por esta especie, comprobando que ella es tolerante a aguas con contaminación ambiental, así como fue señalado por Lacombe (1977b) y Calcagno et al. (1998), actuando como una buena bioindicadora de polución orgánica. En Paripe, un ambiente sin aporte de desechos domésticos, prevaleció la característica fisiológica de Microeuraphia rhizophorae hasta el final del estuario.

La presencia de especies clasificadas como marinas estenohalinas en la estación de mayores valores de salinidad del estuario estudiado pode ser explanada por la proximidad de la desembocadura del estuario con el mar abierto, en la barra Sur del Canal de Santa Cruz. Las especies Amphibalanus venustus, Chthamalus bisinuatus, y Striatobalanus amaryllis, encontradas solamente en las regiones euhalinas y polihalinas fueron también citadas por Castro (1997) para los recifes de Abrollhos, Bahia y por Neves et al. (2007), para la bahía de Paranaguá, Paraná, en Brasil en áreas marinas o próximas al mar. De acuerdo con Young (1993), C. bisinuatus es una especie hartamente registrada apenas para el ambiente marino.

En relación a las especies clasificadas como marinas eurihalinas, Rosa Filho \& Farrapeira-Assunção (1998), encontraran las mismas especies en toda la región estuarina del Canal de Santa Cruz. Otros autores brasileños pesquisando en bahías del Rio de Janeiro (Oliveira 1941, Silva-Brum \& Absalão 1989, Junqueira et al. 2000) también relacionaran estas mismas especies, incluyendo algunas otras que son marinas estenohalinas.

Amphibalanus improvisus parece ser una de las especies más adaptadas a la existencia estuarina, pues tolera salinidades muy bajas (hasta 2) y puede, así mismo, vivir diez meses al año en aguas dulces logrando una adaptación perfecta mediante el ajuste de su medio interno a la dilución externa, sin alterar su actividad normal (Newman 1967). En este estudio esta especie tuve una amplia distribución estuarina, pero fue Microeuraphia rhizophorae que se mostró mejor adaptada a el ambiente salobre, tolerando intensos cambios de salinidad, sendo el único componente verdaderamente estuarino, distribuido por todo el estuario. Estas exigencias ecológicas de E. rhizophorae en cuanto la variación de salinidad y busca por ambientes protegidos en ambientes estuarinos de manglares, fue también encontrada por Oliveira (1941, 1947), Lacombe (1977b), Silva-Brum \& Absalão (1989), Young (1993), Correia (1998), Junqueira et al. (2000) y Farrapeira (2006) en Brasil, y por Southward (1975), Bacon (1976) y Laguna G. (1987), en la región caribeña del Atlántico.

Además, aunque la variación de la salinidad tenga sido considerada el principal factor determinante de la zonación horizontal de los cirrípedos en el estuario del Río Paripe, cabe recordar que la presencia de sustratos adecuados es también esencial y puede haber sido un factor secundario para la ocurrencia de algunas especies. Gordon (1969), Guerrazi (1987) y Silva (1992) destacaran que la disponibilidad de sustratos duros y la competición por el espacio son algunos de los innumerables factores abióticos y bióticos que intervienen para determinar la naturaleza y distribución de la fauna en regiones estuarinas. Un ejemplo disto es la distribución de Amphibalanus venustus, que por se fijar sobre otros animales, tuvieron su distribución limitada a la de los organismos que les han servido de sustrato, en la región polihalina. Las especies Striatobalanus amaryllis y Chthamalus bisinuatus, al contrario, que solamente se localizaron en sustrato rocoso, inexistentes en las estaciones mesohalinas y oligohalinas del estuario estudiado, estaban de acuerdo con sus valencias ecológicas, una vez que son hartamente mencionadas apenas para el ambiente marino (Silva-Brum \& Absalão 1989, Young 1989, 1993, 1994).

La distribución vertical observada en el área estuarina estudiada obedece al padrón de zonación de los organismos en áreas de manglares en las regiones tropicales, al nivel de familia, género y hasta mismo de especie (Rützler 1969, Pérez \& Victoria 1980, Lalana-Rueda 
1986, Inclán 1989, Rivadeneyra 1989, Silva-Brum \& Absalão 1989, Ellison \& Farnsworth 1992, Correia 1996, Silva-Brum \& Martins 1997, Rosa-Filho \& Farrapeira-Assunção 1998, Farrapeira et al. 2000). Hay que ser destacada la ausencia del género Fistulobalanus en la región intermareal inferior en el estuario estudiado. Farrapeira (dados no publicados) había encontrado en 1988 apenas tres ejemplares de la especie $F$. citerosum en esto estuario, en rocas del infralitoral, en la área equivalente a la estación 1 de esta pesquisa, habiendo considerado, en aquella época, que debería ser una colonización reciente, porque esta especie é ampliamente citada para áreas estuarinas del litoral brasileño (Henry 1973, Lacombe \& Monteiro 1974, Henry \& McLaughlin 1975, Young 1994, Correia 1996, 1998, Marques-Silva et al. 2006, Silva et al. 2006, Neves et al. 2007), pero ningún ejemplar de $F$. citerosum fue encontrado en el estuario más recientemente.

En cuanto a la competición por el espacio en el estuario estudiado, se observó un alto grado de epibiosis de las especies incrustantes y sesiles, que, según Garduño (1980), llega a se convertir en un factor limitante; esto fenómeno fue definido por Wahl \& Mark (1999) como una relación de dos o más especies que, por medio de interacciones directas o indirectas, ejercen mayor efecto en las especies asociadas y en la dinámica de las comunidades. Silva-Brum \& Martins (1996) observaron que las especies Amphibalanus amphitrite, A. improvisus, Chthamalus bisinuatus y $C$. proteus además de otras tres especies marinas, son comúnmente encontradas sobre conchas de moluscos. En el estuario estudiado esta epibiosis fue también vista en la zona intermareal, pero ha sido percibida igualmente en las especies Striatobalanus amaryllis, A. reticulatus y A. improvisus en el limítrofe del piso infralitoral. En esta región de la desembocadura del río, fue observado un alto grado de fijación de organismos sobre otros, que parece ser consecuencia de los raros sustratos duros disponibles. La competición de estas tres especies fue curiosa y merece ser acompañada en el transcurso de los años, pues, las dos primeras especies fueron introducidas en el litoral Brasileño (Young 1989, FarrapeiraAssunção 1990) y puede haber una tendencia a el cosmopolitismo en la biota en cuestión, dependiendo de sus valencias ecológicas. En realidad ambas las especies son clasificadas como invasoras en diversos océanos (Ren \& Liu 1978, Kim \& Kim 1980, Yan et al. 2006), llevadas por embarcaciones (Foster \& Willan 1979, Young 1993, Neves et al. 2007, Farrapeira et al. 2007). Amphibalanus reticulatus presenta un comportamiento más agresivo que la otra especie en la colonización de nuevos substratos (Coles et al. 1999), aún más porque tiene una característica fisiológica de eurihalinidad (Southward 1975, Mayer-Pinto \& Junqueira 2003, Neves et al, 2007), demostrando estar perfectamente adaptada a las variaciones de salinidades en el estuario estudiado.

\section{Referencias Bibliográficas}

ACHITUV, Y. 1984. Cirripedes of the mangal ecosystem with emphasis on the hard bottom mangal of Sinai. In Hydrobiology of the mangal (F.D. Por. \& I. Dor, eds.). Dr. W. Junk Publ., Boston, p.71-78.

AL-THAQAFI, K. \& WHITE, K.N. 1991. Effect of shore position and environmental levels on body metal burdens in the barnacle Elminius modestus. Environ. Pollut. 69(2-3):89-104.

BACON, P.R. 1976. The Cirripedia of Trinidad. Stud. Fauna Curaçao Caribbean Is. 50(163):1-55.

BARNES, R.S.K. 1989. What, if anything, is brackish-water fauna? Trans. Roy. Soc. Edin. Earth Sci. 80:235-240.

BRANCO, L.H.Z., MOURA, A.N., SILVA, A.C. \& BITTENCOURTOLIVEIRA, M.C. 2003. Biodiversidade e considerações biogeográficas das Cyanobacteria de uma área de Manguezal do Estado de Pernambuco, Brasil. Acta Bot. Bras. 17(4):585-596.

BREVES-RAMOS, A., LAVRADO, H.P., JUNQUEIRA, A.O.R. \& SILVA, S.H.G. 2005. Succession in rocky intertidal benthic communities in areas with different pollution levels at Guanabara Bay (RJ-Brazil). Braz. Arch. Biol. Technol. 48(6):951-965.

BULGER, A.J., HAYDEN, B.P., MONACO, M.E., NELSON, D.M. \& MCCORMICK-RAY, M.G. 1993. Biologically-based estuarine salinity zones derived from a multivariate analysis. Estuaries 16(2):311-322.

CALCAGNO, J.A., LÓPEZ GAPPA, J. \& TABLADO, A. 1998. Population dynamics of the barnacle Balanus amphitrite in an intertidal area affected by sewage pollution. J. Crustac. Biol. 18(1):128-137.

CASTRO C.B. (Rel.). 1997. Projeto Abrolhos: Desenvolvimento de um plano de ação para a conservação dos recursos marinhos do complexo de Abrolhos. Aspectos ambientais. Instituto Brasileiro de Meio Ambiente e dos Recursos Naturais Renováveis- IBAMA, Salvador, p.1-104.

COLES, S.L., DeFELICE, R.C., ELDREDGE, L.G. \& CARLTON, J.T. 1999. Historical and recent introductions of nonindigenous marine species into Pearl Harbor, Oahu, Hawaiian Islands. Mar. Biol. 135:147-158

CORREIA, M.D. 1996. Recrutamento e colonização de Mytella charruana e Crassostrea rhizophorae (Mollusca: Bivalvia) no complexo estuarinolagunar Mundaú-Manguaba, Alagoas, Brasil. Bol. Estud. Ciênc. Mar. (9):105-117.

CORREIA, M.D. 1998. Fauna associada a troncos de madeira no complexo estuarino-lagunar Mundaú-Manguaba, Alagoas-Brasil. Bol. Estud. Ciênc. Mar. (10):45-64.

DAY, J.H. 1967. The biology of Knysna Estuary, South Africa. In Estuaries (G.H. Lauff). American Association for the Advancement of Science, Washington, p.397-407.

DHN. 2006. Banco nacional de dados oceanográficos; tábuas das marés. Diretoria de Hidrografia e Navegação-DHN, Brasília: https://www.mar. mil.br/ (último acesso em 20/12/2006).

ELLISON, A.M. \& FARNSWORTH, E.J. 1992. The ecology of Belizean mangrove-root fouling communities: patterns of epibiont distribution and abundance, and effects on root growth. Hydrobiologia 247(1-3):87-98.

FARRAPEIRA, C.M.R. 2006. Barnacles (Cirripedia Balanomorpha) of the estuarine region of Recife, Pernambuco, Brazil. Trop. Oceanogr. 34(2):100-119: http://www.propesq.ufpe.br/tropical_oceanography/ volumes/volume_34_2_2006.html. (último acesso em 16/02/2008).

FARRAPEIRA, C.M.R., COELHO-FILHO, P.A. \& SANTOS, M.A. 2000. Zonação vertical da macrofauna bêntica de substratos consolidados do estuário do Rio Paripe (Itamaracá-PE). In MANGROVE 2000 Sustentabilidade de Estuários e Manguezais: Desafios e Perspectivas. Universidade Federal Rural de Pernambuco, Recife, p.1-9.

FARRAPEIRA, C.M.R., MELO, A.V.O.M., BARBOSA, D.F. \& SILVA, K.M.E. 2007. Ship hull fouling in the Port of Recife, Pernambuco. Braz. J. Oceanogr. 55(3):207-221.

FARRAPEIRA-ASSUNÇÃO, C.M. 1990. Ocorrência de Chirona (Striatobalanus) amaryllis Darwin, 1854 e de Balanus reticulatus Utinomi, 1967 (Cirripedia, Balanomorpha) no Estado de Pernambuco. In XVII Congresso Brasileiro de Zoologia. Sociedade Brasileira de Zoologia, Londrina, p.7.

FOSTER, B.A. \& WILLAN, R.C. 1979. Foreign barnacles transported to New Zealand on an oil platform. New Zealand J. Mar. Freshw. Res. 13(1):143-150.

GARDUÑO, M.E. 1980. La fauna sesil intermareal del manglar relacionada con algunos parámetros ambientales de la Laguna de Términos, Campeche, México. In Seminario sobre el Estudio Científico e Impacto Humano en el ecosistema de manglares. UNESCO, Montevideo, p.102-120.

GORDON, C.M. 1969. The apparent influence of salinity on the distribution of barnacle species in Chesapeake Bay (Cirripedia). Crustaceana 16(2):139-142.

GUERRAZI, M.C. 1987. Estudos preliminares sobre a estrutura de uma comunidade de costão rochoso, em mesolitoral, num gradiente de salinidade. In Simpósio sobre Ecossistemas da Costa Sul-Sudeste Brasileira: Síntese dos Conhecimentos. ACIESP, São Paulo, 2:221-232.

GUNTER, G. 1961. Some relation of estuarine organisms to salinity. Limnol. Oceanogr. 6(2):182-190. 
HENRY, D.P. 1973. Descriptions of four new species of the Balanus amphitrite complex (Cirripedia, Thoracica). Bull. Mar. Sci. 23(4):964-1001.

HENRY, D.P. \& McLAUGHLIN, P. 1975. The barnacles of the Balanus amphitrite complex (Cirripedia: Thoracica). Zool. Verh. 141:1-254.

INCLÁN, R.R. 1989. Ecología de la epibiosis en las raíces inmersas de Rhizophora mangle en Bahía de la Ascensión, Quintana Roo, México. Cienc. Mar. 15(1):1-20.

JUNQUEIRA, A.O.R., FALCÃO, A.P.C., MAYER-PINTO, M., VIANA, M.S. \& SILVA, S.H.G. 2000. Spatial and temporal variations on intertidal barnacle abundance in a tropical bay. Nauplius 8(2):195-204.

KIM, I.N. \& KIM, H.S. 1980. Systematic studies on the cirripeds (Crustacea) from Korea. 1. Balanomorph barnacles (Cirripedia Thoracica, Balanomorpha). Korean J. Zool. 23(3):161-194.

LACOMBE, D. 1977a. Anatomia e microanatomia de Balanidae da Baía de Guanabara (Crustacea, Cirripedia). Rev. Bras. Biol. 37(1):151-165

LACOMBE, D. 1977b. Cirripédios da Baía da Ribeira, Angra dos Reis, RJ (Brasil). Publ. Inst. de Pesq. Marinha (109):1-13.

LACOMBE, D. \& MONTEIRO, W. 1974. Balanídeos como indicadores de poluição na Baía de Guanabara. Rev. Bras. Biol. 34(4):633-644

LACOMBE, D. \& RANGEL, E.F. 1978. Cirripédios de Arraial do Cabo, Cabo Frio. Publ. Inst. de Pesq. Marinha (129):1-12.

LAGUNA G., J.E. 1987. Euraphia eastropacensis (Cirripedia, Chthamaloidea), a new species of barnacle from the tropical eastern Pacific: Morphological and electrophoretic comparisons with Euraphia rhizophorae (de Oliveira) from the tropical western Atlantic and molecular evolutionary implications. Pac. Sci. 41(1/4):132-140.

LALANA-RUEDA, R. 1986. Fauna asociada a las raíces de Rhizophora mangle L., en la laguna costera "El Ciego". Rev. Invest. Mar. 7(3):55-65.

MARCHINKO, K.B., NISHIZAKI, M.T. \& BURNS, K.C. 2004. Communitywide character displacement in barnacles: a new perspective for past observations. Ecol. Letters 7(1):114-120.

MARQUES-SILVA, N., BEASLEY, C., GOMES, C., GARDUNHO, D., TAGLIARO, C., SCHORIES, D. \& MEHLIG, U. 2006. Settlement dynamics of the encrusting epibenthic macrofauna in two creeks of the Caeté mangrove estuary (North Brazil). Wetlands Ecol. Manag. 14(1):67-78.

MAYER-PINTO, M. \& JUNQUEIRA, A.O.R. 2003. Effects of organic pollution on the initial development of fouling communities in a tropical bay, Brazil. Mar. Pollut. Bull. 46(11):1495-1503.

MONTEIRO, B.R. \& COELHO FILHO, P.A. 2004. Estrutura populacional do caranguejo-uçá, Ucides cordatus (Linnaeus, 1763) (Crustacea, Decapoda, Ocypodidade), no estuário do Rio Paripe, Itamaracá - Pernambuco. Bol. Tec. Cient. CEPENE 12(1):99-111.

NEVES, C.S., ROCHA, R.M., PITOMBO, F.B. \& ROPER, J.J. 2007. Use of artificial substrata by introduced and cryptogenic marine species in Paranaguá Bay, southern Brazil. Biofouling 23(5):319-30.

NEWMAN, W.A. 1967. On physiology and behavior of estuarine barnacles. J. Mar. Biol. Ass. India 2(3):1038-1066.

NEWMAN, W.A., ROSS, A. 1976. Revision of the balanomorph barnacles; including a catalog of the species. Mem. San Diego Soc. Nat. Hist. 9:1-108.

OLIVEIRA, L.P.H. 1941. Contribuição ao conhecimento dos crustáceos do Rio de Janeiro: Sub-ordem "Balanomorpha" (Cirripedia: Thoracica). Mem. Inst. Oswaldo Cruz 36(1):1-31.

OLIVEIRA, L.P.H. 1947. Distribuição geográfica da fauna e flora da Baía de Guanabara. Mem. Inst. Oswaldo Cruz 45(3):709-734.
PÉREZ, M.E. \& VICTORIA, C.H. 1980. Algunos aspectos de la comunidad asociada a las raíces sumergidas del mangle rojo en dos áreas del Caribe Colombiano. In Seminario sobre el Estudio Científico e Impacto Humano en el ecosistema de manglares. UNESCO, Montevideo, p.215-224.

REN, X. \& LIU, J.Y. 1978. Studies on Chinese Cirripedia (Crustacea). 1. Genus Balanus. Stud. Mar. Sin. 13:119-196.

RIVADENEYRA, R.I. 1989. Ecología de la epibioses en las raíces inmersas de Rhizophora mangle en Bahía de la Ascensión, Quintana Roo, México. Cienc. Mar. 15(1):1-20.

RODRÍGUEZ, G. 1963. The intertidal estuarine communities of Lake Maracaibo, Venezuela. Bull. Mar. Sci. Gulf Caribb. 13(2):197-218.

ROSA FILHO, J.S. \& FARRAPEIRA-ASSUNÇÃO, C.M. 1998. Zonação vertical da macrofauna bentônica dos andares supra litoral e médio litoral dos manguezais da região da Ilha de Itamaracá (Pernambuco - Brasil). Cad. Ômega, Ser. Cienc. Aquat. (4):59-68.

RÜTZLER, K. 1969. The mangrove community, aspects of its structure, faunistics and ecology. In Laguna costeras: Simposio internacional sobre lagunas costeras (origen, dinámica y productividad). UNAM-UNESCO, México, p.515-536.

SILVA, C.A.R., SMITH, B.D. \& RAINBOW, P.S. 2006. Comparative biomonitors of coastal trace metal contamination in tropical South America (N. Brazil). Mar. Environ. Res. 61(4):439-455.

SILVA, C.S. 1992. Distribuição e abundância da fauna macrobentônica do complexo estuarino-lagunar Mundaú-Manguaba (Alagoas-Brasil). Bol. Estud. Ciênc. Mar. (8):45-64.

SILVA-BRUM, I.N. \& ABSALÃO, R.S. 1989. Vertical distribution of barnacles of the intertidal rocky shores of Guanabara Bay, RJ, Brazil. In Coastlines of Brazil (C. Neves, ed.). American Society of Civil Engineers, New York, p.139-153.

SILVA-BRUM, I.N. \& MARTINS, E.M.P. 1996. Epizoísmo de Balanomorpha (Crustacea Cirripedia) em conchas de Mollusca, Baía da Guanabara, RJ, Brasil. Bolm. Mus. Nac. N. S. (370):1-10.

SILVA-BRUM, I.N. \& MARTINS, E.M.P. 1997. Seasonal variation of the intertidal community of Balanomorpha of Guanabara Bay, Rio de Janeiro, Brazil (Crustacea Cirripedia). Rev. Bras. Biol. 57(2):275-288.

SOUTHWARD, A.J. 1975. Intertidal and shallow water Cirripedia of the Caribbean. Stud. Fauna Curaçao Carib. Is. 46(150):1-53.

WAHL, M. \& MARK, O. 1999. The predominantly facultative nature of epibiosis: experimental and observational evidence. Mar. Ecol. Progr. Ser. 187:59-66.

YAN, T., YAN, W.X., DONG, Y., WANG, H.J., YAN, Y. \& LIANG, G.H. 2006. Marine fouling of offshore installations in the northern Beibu Gulf of China. Int. Biodeterior. Biodegrad. 58(2):99-105.

YOUNG, P.S. 1989. Establishment of an Indo-Pacific barnacle in Brazil. Crustaceana 56(2):212-214.

YOUNG, P.S. 1991. The superfamily Coronuloidea (Cirripedia: Balanomorpha) from Brazilian coast, with redescription of Stomatolepas species. Crustaceana 61(2):189-212.

YOUNG, P.S. 1993. The Verrucomorpha and Chthamaloidea from the Brazilian coast (Crustacea: Cirripedia). Rev. Bras. Biol. 53(2):247-253.

YOUNG, P.S. 1994. Superfamily Balanoidea Leach (Cirripedia, Balanomorpha) from the Brazilian coast. Bolm. Mus. Nac. N. S. (356):1-36.

Recibido en $28 / 04 / 08$ Versión revisada en $21 / 05 / 08$ Publicado en 01/07/08 


\section{Apéndice}

Clave dicotómica de identificación de los cirrípedos del estuario del Río Paripe:

1. Placas parietales sólidas; base membranosa o calcárea...... .2

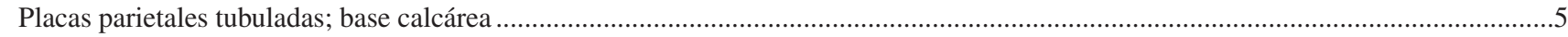

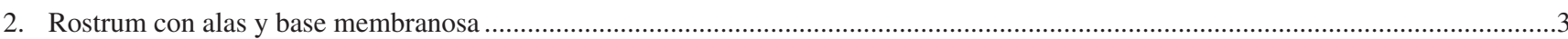

- Rostrum con rádios estrechos, generalmente inclinado en dirección a la carina; placas carino-laterales estrechas y base calcárea (generalmente de grande dimensión)

Striatobalanus amaryllis

3. Escudo más alto que ancho; articulación escuto-tergal recta; mandíbula tridentada

Microeuraphia rhizophorae

- Escudo más ancho que alto; articulación escuto-tergal sinuosa; mandíbula con cuatro dientes

.4

4. Concha cónica con costillas verticales cerca de la base; placas parietales distintas; articulación escuto-tergal con ligera sinuosidad; internamente; cerdas bidenticuladas del cirro II con guardas basales Chthamalus proteus

- Concha plana, lisa o erodida; placas parietales generalmente indistintas, fusionadas; articulación escuto-tergal con sinuosidad dupla; cerdas bidenticuladas del cirro II sin guardas basales... .Chthamalus bisinuatus

5. Concha blanca con líneas verticales coloridas, violetas o marrones en las placas parietales ................................................6

- Concha blanca con líneas verticales hialinas o ligeramente coloridas y estrechas en las placas parietales

6. Concha con líneas verticales que forman grupos de 2 a 4 líneas en cada placa parietal; radii anchos, cúspide horizontal casi paralela a la base; apófisis del tergo fasciolado, más ancho que largo; labrum multidenticulado ...Amphibalanus amphitrite

- Concha con líneas verticales y transversales formando un padrón reticulado; radii delgados, cúspide muy oblicua; apófisis del tergo con surco longitudinal, más largo que ancho; labrum simples

Amphibalanus reticulatus

7. Concha blanca con líneas verticales hialinas; radii delgados, cúspide muy oblicua, redondeada y lisa; apófisis del tergo con surco longitudinal, más largo que ancho, con final obtuso o redondeado; labrum multidenticulado..... Amphibalanus improvisus

- Concha blanca con líneas verticales hialinas o levemente coloridas; radii anchos, espesas, cúspide rugosa, moderadamente oblicua, redondeada y lisa; apófisis del tergo fasciolado, más ancho que largo, con final truncado; labrum simple.

Amphibalanus venustus 